\title{
INSECTICIDAL ACTIVITIES OF ABROMA AUGUSTA (L.) CHLOROFORM AND METHANOL EXTRACTS AGAINST TRIBOLIUM CASTANEUM (HERBST) ADULTS
}

\author{
Omar Ali Mondal ${ }^{1}$, Esarul Haque ${ }^{2}$, Jahurul Haque ${ }^{1}$ and Ataur Rahman Khan ${ }^{1 *}$ \\ ${ }^{1}$ Department of Zoology, University of Rajshahi, Rajshahi-6205, Bangladesh, \\ ${ }^{2}$ Department of Zoology, Premtali Degree College, Godagari, Rajshahi, Bangladesh
}

\begin{abstract}
The chloroform and methanol extracts of the leaves, root wood, stem bark, stem wood and seeds of Abroma augusta (L.) (Ulatkambal) were tested against Tribolium castaneum (Herbst) adults through residual film assay. The seed extracts $\left(\mathrm{CHcl}_{3}\right.$ and $\left.\mathrm{MeOH}\right)$ were found to offer the highest mortality of the beetles and the $\mathrm{LD}_{50}$ values were $3046.083,247.9217$ and $75.96001 \mu \mathrm{g} / \mathrm{cm}^{2}$ and $6598.793,340.4855$ and 113.6461 $\mu \mathrm{g} / \mathrm{cm}^{2}$ for the $\left(\mathrm{CHCl}_{3}\right.$ and $\left.\mathrm{MeOH}\right)$ extracts for 30min, 24 and $48 \mathrm{~h}$ of exposures respectively. The root wood extracts gave $\mathrm{LD}_{50}$ values of 1127.785, 312.5822 and $146.3708 \mu \mathrm{g} / \mathrm{cm}^{2}$ and 1689.468, 449.8259 and $134.9692 \mu \mathrm{g} / \mathrm{cm}^{2}$ for the $\left(\mathrm{CHCl}_{3}\right.$ and $\left.\mathrm{MeOH}\right)$ extracts for $30 \mathrm{~min}, 24 \mathrm{and}$ $48 \mathrm{~h}$ of exposures respectively. These were followed by the leaf $\left(\mathrm{CHCl}_{3}\right.$ and $\left.\mathrm{MeOH}\right)$ extracts that gave $\mathrm{LD}_{50}$ values of $3466.807,450.3049$ and $175.7438 \mu \mathrm{g} / \mathrm{cm}^{2}$ and 4654.238, 1010.538 and $333.7202 \mu \mathrm{g} / \mathrm{cm}^{2}$ for the $\left(\mathrm{CHCl}_{3}\right.$ and $\left.\mathrm{MeOH}\right)$ extracts for $30 \mathrm{~min}$, 24h and 48h of exposure respectively. The stem bark extracts $\left(\mathrm{CHCl}_{3}\right.$ and $\left.\mathrm{MeOH}\right)$ gave $\mathrm{LD}_{50}$ values of 3295.859, 1137.558 and $363.1539 \mu \mathrm{g} / \mathrm{cm}^{2}$ and $3717.851,566.2215$ and $230.7044 \mathrm{\mu g} / \mathrm{cm}^{2}$ for the $\left(\mathrm{CHCl}_{3}\right.$ and $\left.\mathrm{MeOH}\right)$ extracts for $30 \mathrm{~min}, 24$ and $48 \mathrm{~h}$ of exposure respectively. The stem wood extracts $\left(\mathrm{CHCl}_{3}\right.$ and $\mathrm{MeOH}$ ) of $\mathrm{A}$. augusta did not show any activity against the beetles. According to the intensity of activity observed through mortality of the adult beetles the potentiality of the chloroform extracts could be arranged in a descending order of seeds $>$ root wood $>$ leaf $>$ stem bark and for the methanol extracts, seed $>$ root wood $>$ stem bark > leaf.
\end{abstract}

Key wards: Abroma augusta, Insecticidal activity, Chloroform and methanol extracts, Tribolium castaneum.

সারাংশ: Tribolium castaneum (Herbst) এর পূর্ণাংগ পোকার উপর Abroma augusta (L.) (উলটকম্বল) -এর পাতা, মূনের কাঠ, কান্ডের বাকল, কান্ডের কাঠ ও বীজের ক্লোরোফর্ম ও মিথানল নির্যাসের বিষক্রিয়া পর্যবেক্ষণ করা হয়। বীজের ক্লোরোফর্ম ও মিথানল নির্যাস ৩০ মিনিট, ২৪ ঘন্টা ও 8b- ঘন্টায় পোকাগুলির উপর সবচেয়ে বেশী কার্যকারিতা প্রদর্শন করে যার LD মূলের কাঠের ক্লোরোফর্ম ও মিথানল নির্यালের ক্ষেত্রে LD $_{50}$ ছিল যথাক্রমে $1127.785,312.5822$ ও $146.3708 \mu \mathrm{g} / \mathrm{cm}^{2}$ এবং 1689.468, 449.8259 ও $134.9692 \mu \mathrm{g} / \mathrm{cm}^{2}$ । পাতার ক্লোরোফর্ম ও মিথানল নির্যালের ক্ষেত্রে যথাক্রমে 3466.807, 450.3049 ও $175.7438 \mu \mathrm{g} / \mathrm{cm}^{2}$ এবং 4654.238, 1010.538 ও $333.7202 \mu \mathrm{g} / \mathrm{cm}^{2}$ কার্যকারিতা প্রদর্শন করে। কান্ডের বাকলের ক্লোরোফর্ম ও মিথানল নির্যাসের ক্ষেত্রে 3295.859, 1137.558 ও $363.1539 \mu \mathrm{g} / \mathrm{cm}^{2}$ এবং 3717.851, 566.2215 ও $230.7044 \mu \mathrm{g} / \mathrm{cm}^{2}$ কার্যকারিতা প্রদর্শন করে। কান্ডের কাঠের কোন নির্যাস Tribolium castaneum -এর উপর কোন কার্যকারিতা প্রদর্শন করে নাই। কার্যকারিতার মাত্রা অনুসারে ক্লোরোফর্ম নির্যাসগুলিকে যথাক্রমে বীজ>মূলের কাঠ>পাতা>কান্ডের বাকল এবং মিথানল নির্যাসগুলিকে বীজ>মূলের কাঠ>কান্ডের বাকল>পাতা ক্রমানুসারে সাজানো যায়।

\section{Introduction}

Botanical pesticides, as an alternative to the conventional chemical agents for insect control are now-a-days very popular among the pest control experts. They are more readily biodegradable and therefore, are less likely to contaminate the environment. Moreover, natural compounds break down readily in soil and are not stored in plant or animal tissues. The use of phytochemicals as powder, oil and extracts for the control of stored-product insect pests has much agricultural importance and recently has received much more attention because these insecticidal compounds are safer than synthetic pesticides, cheaper and can easily be obtained from plants with less sophisticated methods.

Abroma augusta L. (Sterculiaceae), commonly known as ulatkambal, is a large spreading bushy shrub with fibrous barks and irritant hairs. The branches and branch lets are downy. When freshly cut the root produces a thick gummy substance. The root bark is tasteless, slimy, odorless and tough. When soaked in cold water for 3-4 days, the bark produces slimy mucilage which can be extracted (Anonymous 2006, Nandkarni 2002).

Being a medicinal plant, A. augusta contains antipathogenic properties and almost all the parts of the plant are used in the treatment of different diseases. Insecticidal activities of the plant have been demonstrated by a number of recent workers viz., Prajapati et al. (2003), Nanda (1997), Halim (2003), Hanif et al. (2010), Kirtikar and Basu (1918, 1999), Nandkarni (2002), Rahamtullah et al. (2010).

The rust-red flour beetle, Tribolium castaneum (Herbst) is a major pest of a wide range of stored commodities. The insecticidal activities of $A$. augusta extracts have been attempted and the investigation has been designed to evaluate the efficacy of the plant parts as a possible source of potential secondary metabolites to be used as environment-friendly pest control agents against $T$. castaneum. 


\section{Materials and Methods}

Preparation of plant materials for extraction: The experimental plant (A. augusta) was collected from the campus of the University of Rajshahi, Bangladesh. After drying under shade the plant materials, viz. leaves, root wood, seeds, stem bark and stem-wood were powdered in a grinder separately.

Chemical extraction from the plant materials: Chloroform and methanol were selected as solvents to extract different parts of $A$. augusta separately. The ground dried materials were extracted with sufficient amounts of chloroform $(500 \mathrm{~g} \times 1500 \mathrm{ml} \times 3$ times $)$ for each of the items. Separate extracts were collected by the cool method after 72 hours of plunging for each of the materials. Extracts, thus obtained, were subjected to filtration and evaporation of the solvents. The residue were left and kept in a refrigerator after proper labeling.

\section{Application of doses:}

Through an ad hoc experiment a general concentration for the extracts was selected as $10 \mathrm{mg} / 2 \mathrm{ml}$ as the stock solution for surface film application to make other successive doses by serial dilution to give 1040, 780, 520, 260, 130 and $65 \mu \mathrm{g} / \mathrm{cm}^{2}$ concentrations. The application of the doses was made by the residual film method (Busvine, 1971). For each dose 1ml of the extract was dropped on a Petri dish (70 mm diam.) in such a way that it made a uniform film over the Petri dish. Then the Petri dishes were air-dried and 10 beetles (3-5-day old) were released in each of the Petri dishes with three replicates. A control batch was also maintained with the same number of insects after preparing the Petri dish by applying and evaporating the solvent only. Mortality data were recorded for 30min, 24h and 48h postexposure.

\section{Statistical Analyses}

The mortality of the beetles was corrected by the Abbott's (1925) formula:

$\mathrm{P}_{\mathrm{r}}=\frac{\mathrm{P}_{0}-\mathrm{P}_{\mathrm{c}}}{100-\mathrm{P}_{\mathrm{C}}} \times 100$; Where, $\mathrm{P}_{\mathrm{r}}=$ Corrected mortality (\%);

$\mathrm{P}_{\mathrm{o}}=$ Observed mortality (\%) and $\mathrm{P}_{\mathrm{C}}=$ Control mortality (\%).

Data were subjected to statistical analyses according to Finney (1947) and Busvine (1971). The experiment was conducted at a room temperature of $30 \pm 2^{\circ} \mathrm{C}$.

\section{Results and Discussion}

The results have been presented in Tables 1 and 2 for the mortality recorded and have been shown in Fig.1 and 2. The seed extracts were found to offer the highest mortality of the beetles for both the solvents. According to the degree of activity observed through mortality of the adult beetles the potentiality of the chloroform extracts could be arranged in a descending order of seed $>$ root wood $>$ leaf $>$ stem bark extracts and of the methanol extracts, seed $>$ root wood $>$ stem bark $>$ leaf extracts.

Table 1 Dose-mortality effects of $A$. augusta extracts $\left(\mathrm{CHCl}_{3}\right)$ against $T$. castaneum adults.

\begin{tabular}{|c|c|c|c|c|c|c|}
\hline \multirow{2}{*}{ 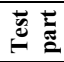 } & \multirow{2}{*}{ Exposure period } & \multirow{2}{*}{$L_{50}$ value $\left(\mu \mathrm{g} / \mathrm{cm}^{2}\right)$} & \multicolumn{2}{|c|}{ 95\% Conf. limits } & \multirow{2}{*}{ Regression equation } & \multirow{2}{*}{$\begin{array}{c}\chi^{2-} \text { Value } \\
\text { (df) }\end{array}$} \\
\hline & & & Lower limit & Upper limit & & \\
\hline \multirow{3}{*}{ 荧 } & $30 \mathrm{~min}$. & 3466.807 & 434.898 & 27635.810 & $\mathrm{Y}=2.112+0.816 \mathrm{X}$ & $0.199(3)$ \\
\hline & $24 \mathrm{~h}$ & 450.305 & 280.002 & 724.190 & $\mathrm{Y}=1.863+1.182 \mathrm{X}$ & $1.549(3)$ \\
\hline & $48 \mathrm{~h}$ & 175.744 & 129.568 & 238.377 & $\mathrm{Y}=1.099+1.738 \mathrm{X}$ & $5.069(3)$ \\
\hline \multirow{3}{*}{ 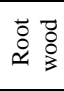 } & $30 \mathrm{~min}$. & 1127.785 & 465.277 & 2733.641 & $\mathrm{Y}=1.668+1.092 \mathrm{X}$ & $0.447(3)$ \\
\hline & $24 \mathrm{~h}$ & 312.582 & 207.490 & 470.902 & $\mathrm{Y}=2.002+1.202 \mathrm{X}$ & $0.124(3)$ \\
\hline & $48 \mathrm{~h}$ & 146.371 & 98.901 & 216.626 & $\mathrm{Y}=1.936+1.415 \mathrm{X}$ & $2.483(3)$ \\
\hline \multirow{3}{*}{ 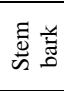 } & $30 \mathrm{~min}$. & 3295.859 & 889.985 & 12205.470 & $\mathrm{Y}=0.197+1.365 \mathrm{X}$ & $0.229(3)$ \\
\hline & $24 \mathrm{~h}$ & 1137.558 & 538.928 & 2401.138 & $\mathrm{Y}=1.838+1.035 \mathrm{X}$ & $0.529(3)$ \\
\hline & $48 \mathrm{~h}$ & 363.154 & 233.226 & 565.463 & $\mathrm{Y}=2.182+1.100 \mathrm{X}$ & $1.219(3)$ \\
\hline \multirow{3}{*}{$\underset{\Phi}{\mathscr{J}}$} & $30 \mathrm{~min}$. & 3046.083 & 370.990 & 25010.390 & $\mathrm{Y}=2.258+0.787 \mathrm{X}$ & $0.158(4)$ \\
\hline & $24 \mathrm{~h}$ & 247.922 & 131.750 & 466.527 & $\mathrm{Y}=2.895+0.879 \mathrm{X}$ & $0.337(4)$ \\
\hline & $48 \mathrm{~h}$ & 75.960 & 54.368 & 106.128 & $\mathrm{Y}=2.364+1.402 \mathrm{X}$ & $3.868(4)$ \\
\hline
\end{tabular}

Table 2 Dose-mortality effects of $A$. augusta extracts $\left(\mathrm{CH}_{3} \mathrm{OH}\right)$ against $T$. castaneum adults.

\begin{tabular}{|c|c|c|c|c|c|c|}
\hline \multirow{2}{*}{ 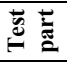 } & \multirow{2}{*}{ Exposure period } & \multirow{2}{*}{$\mathrm{LD}_{50}$ value $\left(\mu \mathrm{g} / \mathrm{cm}^{2}\right)$} & \multicolumn{2}{|c|}{ 95\% Conf. limits } & \multirow{2}{*}{ Regression equation } & \multirow{2}{*}{$\begin{array}{c}\chi^{2} \text {-Value } \\
\text { (df) }\end{array}$} \\
\hline & & & Lower limit & Upper limit & & \\
\hline \multirow{3}{*}{$\stackrel{\widetilde{U}}{\mathbb{U}}$} & $30 \mathrm{~min}$. & 4654.238 & 496.941 & 43590.610 & $\mathrm{Y}=1.481+0.959 \mathrm{X}$ & $0.183(3)$ \\
\hline & $24 \mathrm{~h}$ & 1010.538 & 376.168 & 2714.704 & $\mathrm{Y}=2.293+0.901 \mathrm{X}$ & $1.721(3)$ \\
\hline & $48 \mathrm{~h}$ & 333.720 & 204.656 & 544.177 & $\mathrm{Y}=2.434+1.017 \mathrm{X}$ & $1.662(3)$ \\
\hline \multirow{3}{*}{ 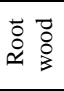 } & $30 \mathrm{~min}$. & 1689.468 & 591.451 & 4825.927 & $\mathrm{Y}=1.057+1.221 \mathrm{X}$ & $0.242(3)$ \\
\hline & $24 \mathrm{~h}$ & 449.826 & 259.868 & 778.641 & $\mathrm{Y}=2.288+1.022 \mathrm{X}$ & $0.243(3)$ \\
\hline & $48 \mathrm{~h}$ & 134.969 & 82.772 & 220.082 & $\mathrm{Y}=2.535+1.157 \mathrm{X}$ & $3.755(3)$ \\
\hline \multirow{3}{*}{ 苞层 } & $30 \mathrm{~min}$. & 3717.851 & 717.054 & 19276.680 & $\mathrm{Y}=1.323+1.030 \mathrm{X}$ & $0.779(3)$ \\
\hline & $24 \mathrm{~h}$ & 566.222 & 358.554 & 894.166 & $\mathrm{Y}=1.993+1.092 \mathrm{X}$ & $0.761(3)$ \\
\hline & $48 \mathrm{~h}$ & 230.704 & 156.868 & 339.295 & $\mathrm{Y}=1.225+1.597 \mathrm{X}$ & $5.414(3)$ \\
\hline \multirow{3}{*}{ ڤัँ } & $30 \mathrm{~min}$. & 6598.793 & 285.020 & 152775.800 & $Y=2.450+0.668 X$ & $0.220(4)$ \\
\hline & $24 \mathrm{~h}$ & 340.486 & 161.627 & 717.271 & $\mathrm{Y}=2.824+0.860 \mathrm{X}$ & $0.207(4)$ \\
\hline & $48 \mathrm{~h}$ & 113.646 & 77.444 & 166.772 & $Y=2.558+1.188 X$ & $1.013(4)$ \\
\hline
\end{tabular}




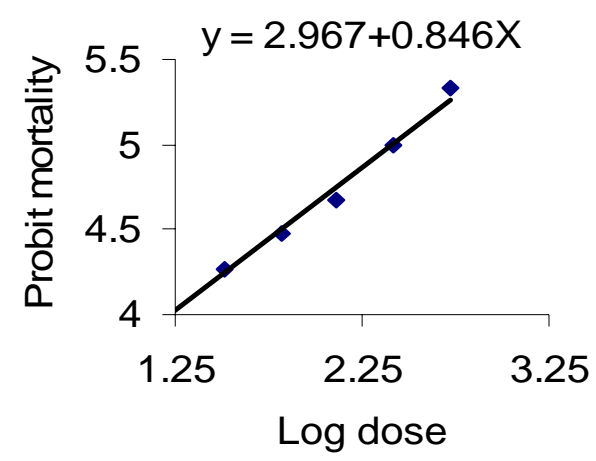

A

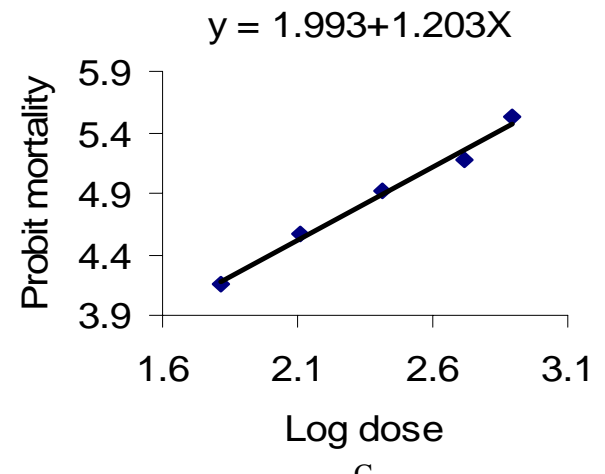

$\mathrm{C}$
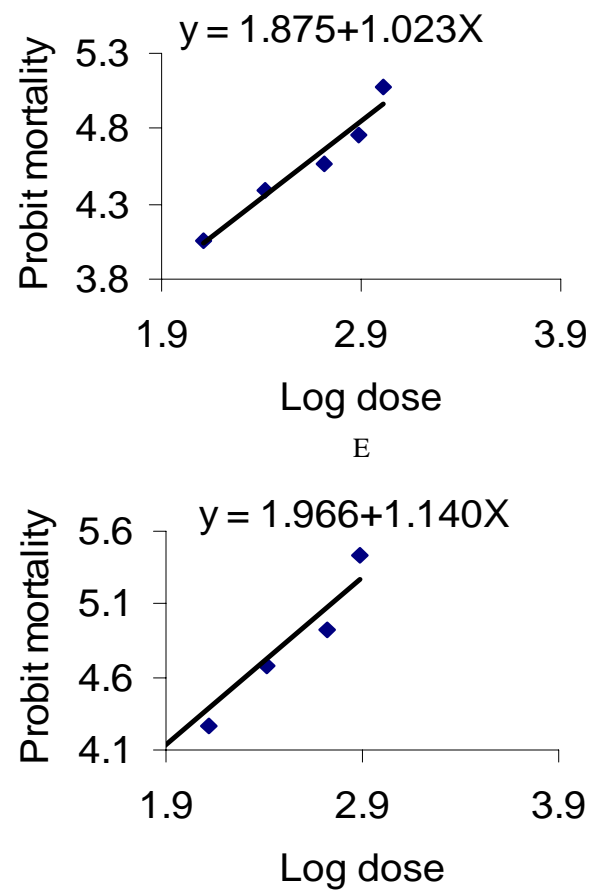

G

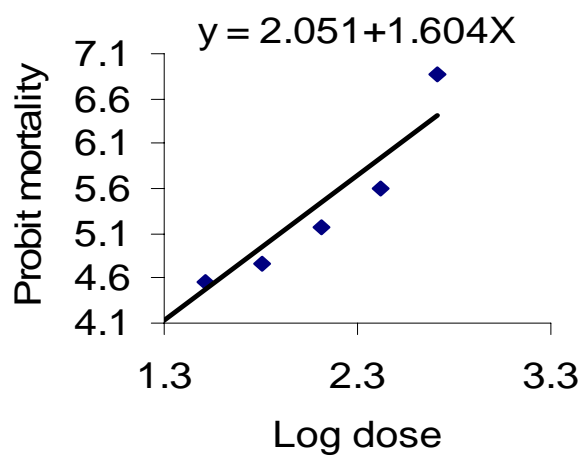

B

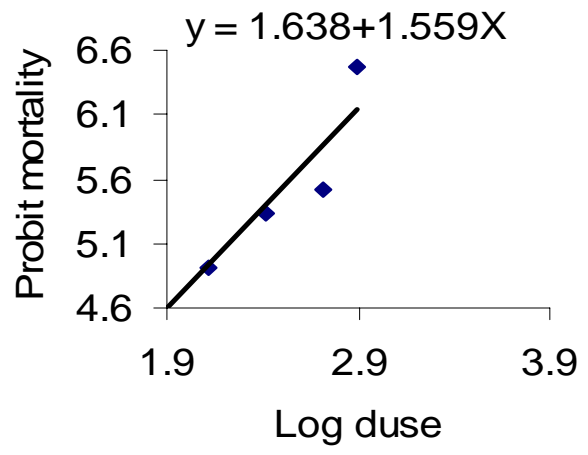

$\mathrm{D}$

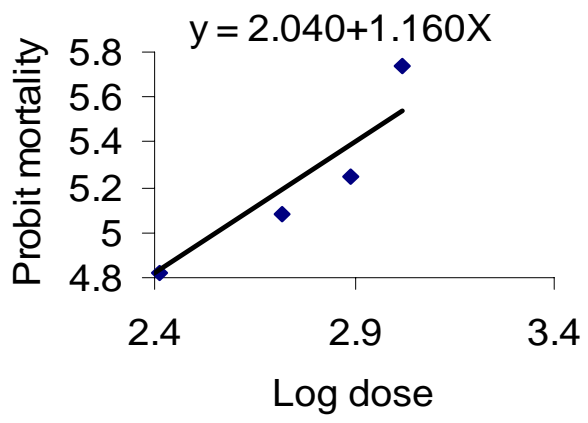

$\mathrm{F}$

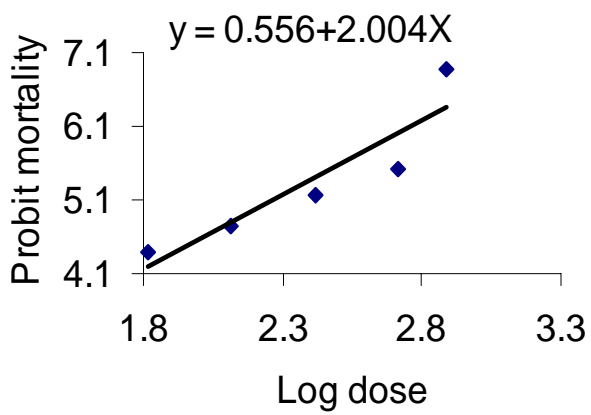

$\mathrm{H}$

Fig.1 Probit mortality regression lines of the chloroform extracts of A. augusta: A- Seed/ 24 h; B- Seed/ 48 h; C- root wood/ 24 h; D- root wood/ $48 \mathrm{~h}$; E- stem bark/ $24 \mathrm{~h}$; F- stem bark/ 48 h; G- leaf/ $24 \mathrm{~h}$ and H- leaf/ $48 \mathrm{~h}$ of exposure against $T$. castaneum adults. 


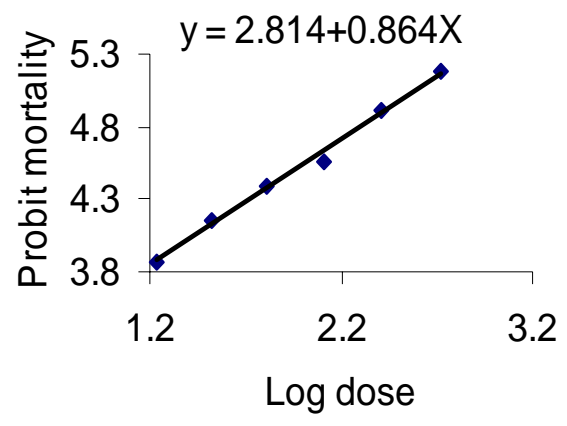

A

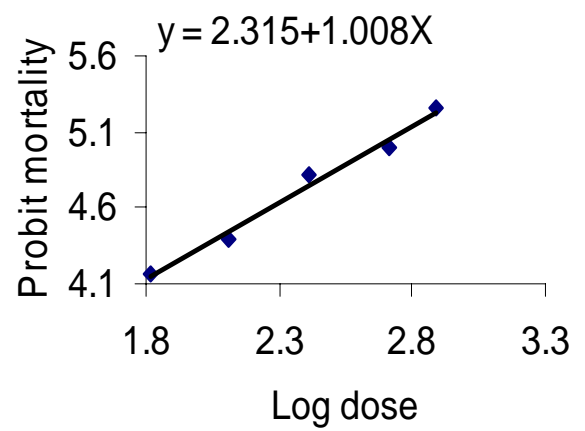

C

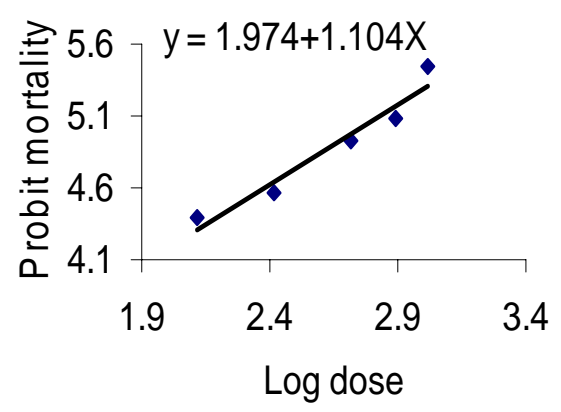

$\mathrm{E}$

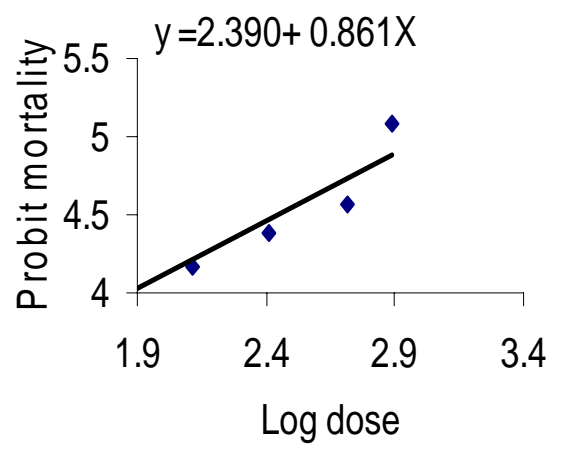

G

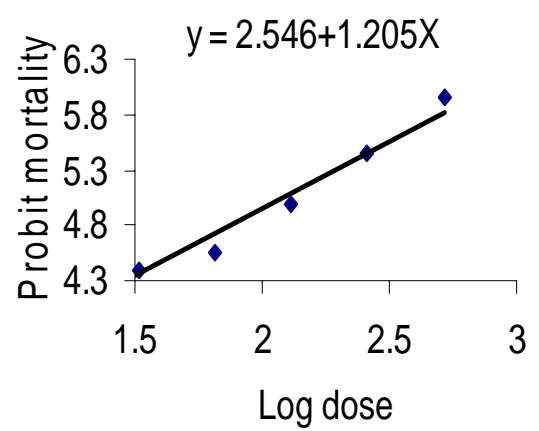

B
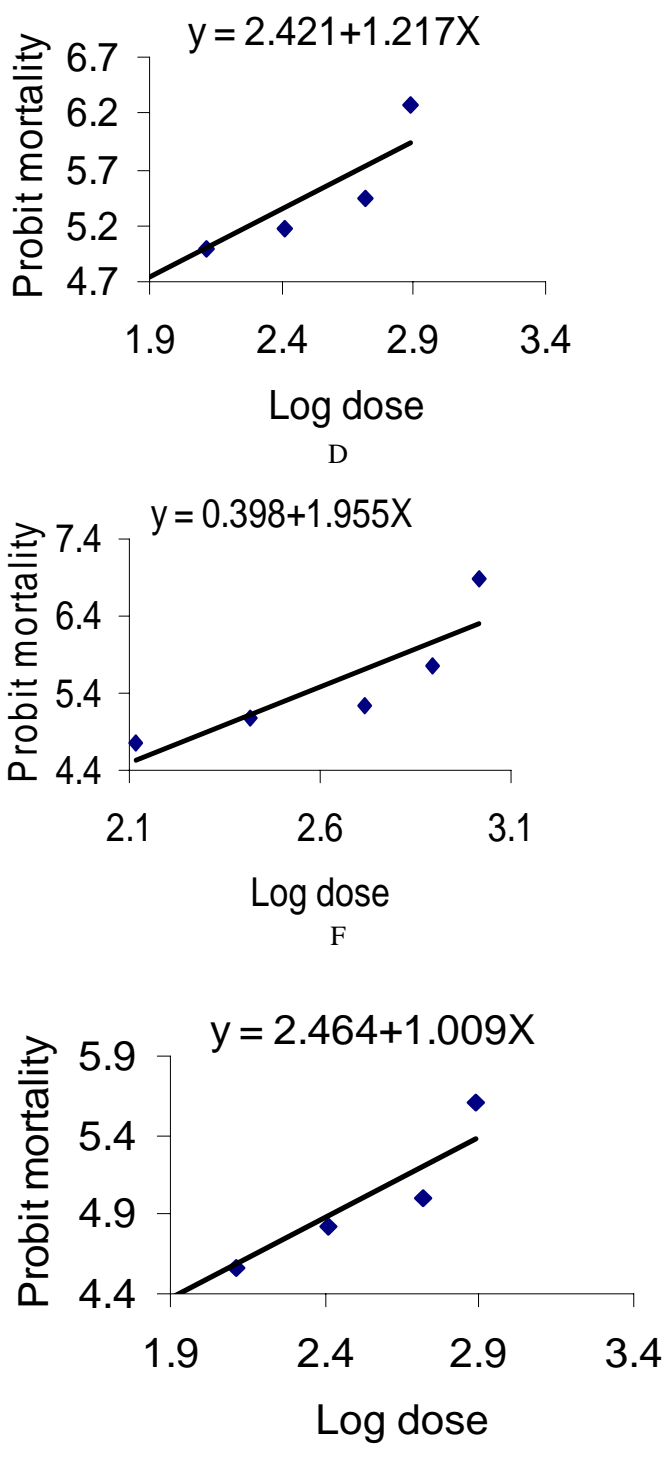

$\mathrm{H}$

Fig. 2 Probit mortality regression lines of the methanol extracts of A. augusta: A- Seed/ 24 h; B- Seed/ 48 h; C- root wood/ 24 h; D- root wood/ $48 \mathrm{~h}$; E- stem bark/ 24 h; F- stem bark/ 48 h; G- leaf/ 24 h and H- leaf/ 48 h of exposure against $T$. castaneum adults. 
The seed extracts (of both the solvents) offered the highest mortality of $T$. castaneum. However, the comparatively higher doses of the root wood, stem bark and leaf extracts indicate weaker action. The test extracts produced mortality within $30 \mathrm{~min}$ of application just to prove their acute toxicity. The seed, root wood and stem bark extracts were found to possess bioactive potential(s) with comparatively higher insecticidal activity but the stem bark extract was comparatively mild in action followed by the leaf extract. The stem wood extract did not show any activity against $T$. castaneum. The present results support the previous work of Naqvi and Parveen (1991) who observed that the seed extract of A. augusta showed a remarkable insecticidal activity against $T$. castaneum.

The present results clearly demonstrate that both the chloroform and methanol extractives from various parts of $A$. augusta have significant insecticidal activities. These results are in agreement with similar works of Krishnamurti and Rao (1944), Su et al. (1972), Sangapa (1977) and Rao et al. (2010). Abdullah et al. (2011) assessed the mortality and repellency of the chloroform extracts of different parts of Urena sinuata on $T$. castaneum adults.

\section{Conclusion}

A perusal of the data reveal that $A$. augusta extracts can be used as insecticidal compounds in the grain and cereal stores to manage the population of $T$. castaneum. The results also seem to be encouraging when there is a greater need for environment-friendly pesticides than ever before. The overall assessment of toxicity of $A$. augusta extracts are very much promising and their efficacy on stored grain pests might have future to be used as a control agent or tool. It may open its possibility as a control agent for the insect pests as well. However, much more comprehensive investigation is needed in this area.

\section{Acknowledgement}

The authors remain grateful to the Chairman, Department of Zoology, University of Rajshahi, Bangladesh, for kindly supplying the required laboratory facilities.

\section{References}

Abbott, WS. 1925. A method of computing the effectiveness of an insecticide. J. Econ. Ent. 18: 265-267.

Abdullah M, PK. AK, Saleh DKMA, Khan AR, Islam R and Islam N. 2011. Insecticidal and repellent activities of the chloroform extracts of Urena sinuata L. against Tribolium castaneum (Herbst) adults. Univ. j. zool. Rajshahi Univ. 30:25-28.

Anonymous. 2006. "The wealth of India" dictionary of Indian raw materials and industrial products. The council of scientific and industrial research. NISCAIR press publisher, New Delhi. 222pp.

Busvine, JR. 1971. A critical review of the techniques for testing insecticides. Commonwealth Agricultural Bureaux, London. 345 pp.

Das S, Datta R and Nandy S. 2012. Phytochemical screening and evaluation of anti-inflammatory activity of methanolic extract of Abroma augusta Linn. Asian Pacific J. Trop.Dis.Vol. - 114-117.

Finney, DJ. 1947. Probit analysis: a statistical treatment of the sigmoid response curve. Cambridge University Press, London. 333pp.

Gupta B, Nayak S and Solanki S. 2011. Der Pharmacia Sinica. 2(4):253-261.

Halim, E. 2002. Indian J. Clin. Biochem. 17 (2): 33-43.

Hanif A, Sh H, Agarwal B, Mohammed R and Rowank J. 2010. A journal of plants, people and applied research Ethnobotany research and applications (8): 61-74.

Kirtikar KR and Basu BD. 1918. Indian Medicinal Plants. Sudhindra Nath Basu publishers, Bahadur Ganj, Allahabad. pp. 210-211.

Kritikar KR and Basu BD. 1999. Textbook of "Indian Medicinal Plants", vol. 1(2nd edn.). Surendra Nath Basu Publishers, Bahadur Ganj, Allahabad. 379pp.

Krishnamurti B and Rao BS. 1944. Results of certain experiments and observations carried out recently in the matter of control of insect pests of food grain in storage. Mysore Agric. 22(3): 91-101.

Mishra S, Rao C and Ojha S. 2010. Indian J. Pharmaceu. Sci. \& research. 4(2):99-104.

Nanda, UK. 1997. Clinical evaluation of non-hormonal drug minofil in the management of post menopausal syndrone Maturitas, 27:215-215.

Nandkarni, KM. 2002. Indian materia medica, vol. I. Bombay Popular Prakashani, Mumbai.

Naqvi SNH and Parveen. 1991. Toxicity and Residual Effect of Nerium indicum Crude Extract as Compared With Coopex Against Adults of Tribolium castaneum. Pak. J. Ethnol. 6:35-44.

Prajapati ND, Purohit SS, Sharma AK and Kumar T. 2003. A handbook of medicinal plants. A complete source book. Agrobios (India) Publisher. Jodhpur. pp. 337-344.

Rao L, Dathi S and Atmakuri. 2010. J. Pharm. \& Res. 3(1): 109-113.

Rahmatullah M, Bachar C, Rahman S and Jahan R. 2010. Advancse, in natural and applied science. 4(2): 163-173.

Sangappa, HK. 1977. Effectiveness of oils surface protectants against the bruchid, Callosobuchus chinensis Linn. Infestation on red grain. Mysore J. Agric. Sci. 11: 391-397.

Su HCF, Speir RD and Mahany PG. 1972. Citrus oils as protectants of blackeyed peas against cowpea weevils: laboratory evaluations. J. Econ. Ent. 65: 1433-1436.

Manuscript received on 9 July 2013 and revised on 9 November 2013 